\title{
Téoros
}

Revue de recherche en tourisme

\section{Les jardins du tourisme}

\section{Gérard Beaudet et Alexander Reford}

Volume 18, numéro 1, printemps 1999

Les jardins du tourisme

URI : https://id.erudit.org/iderudit/1072294ar

DOI : https://doi.org/10.7202/1072294ar

Aller au sommaire du numéro

Éditeur(s)

Université du Québec à Montréal

ISSN

0712-8657 (imprimé)

1923-2705 (numérique)

Découvrir la revue

Citer ce document

Beaudet, G. \& Reford, A. (1999). Les jardins du tourisme. Téoros, 18(1), 3-4. https://doi.org/10.7202/1072294ar d'utilisation que vous pouvez consulter en ligne.

https://apropos.erudit.org/fr/usagers/politique-dutilisation/ 


\section{LES JARDINS DU TOURISME}

\section{Gérard Beaudet et Alexander Reford}

Le jardin est d'emblée un ailleurs, un lieu d'évasion. quand bien mêmé serait-il aménagé sur le pas de la porte de la résidence. Il inscrit quiconque le fréquente dans une duré et des rythmes qui lui sont propres. Certes il fallait, pour que le jardin devienne touristique, qu'advienne le tonriste. Or, l'invention du tourisme, qu'on situe à la fin du XVIIF siècle, est relativement récente. Stricto Sensu, le jardin touristique, si tant est qu'il existe sur ce mode exclusif, est done une création contemporaine. En revanche, les jardins du tourisme n'appartiennent pas à proprement parler à l'áge du tourismé. Ils répondent d'un univers culturel aussi ancien que l'établissement humain. S'étonnera-t-on que les jardins du tourisme soient souvent des jardins historiques?

Depuis près de deux décennies, au Québec comme un peu partout en Occident, l'art des jardins suscite un intérểt considérable. Cet engonement s'exprime dans les espaces de proximité et du quotidien, comme en témoigne l'essor de l'horticulture ornementale. Mais il se traduit également par une entrée remarquée du jardin dans le champ du patrimoine et du tourisme.

Les jardins comptent au nombre des sites touristiques les plus achalandés. En Europe, leur fréquentation surpasse celle des musées, des centres culturels et des galeries d'art. En Amérique du Nord, leur popularité connaît une forte croissance. Cet engouement a contribué à la mise en valeur de jardins anciens et à la création d'autres jardins. Les responsables de nombreux sites historiques ont mis en valeur les jardins anciens qu'on y trouvait — Les Jardins de Métis à Grand Métis, le Domaine Joly-de Lotbinière, la maison Henry-Stuart à Québec, le Domaine Howard à Sherbrooke, etc. — ou ont aménagé des jardins pour étayer leur produit et élar'gir leur clientèle, par exemple la roseraie du Témiscouata au Fort Ingall à Cabano. Dans l'Est du Canada, plusieurs jardins publics ont par ailleurs été créés au cours des années $1990:$ le jardin botanique du Nouveau-Brunswick, les Grands Jardins de Normandin, Kingsbrae Horticultural Gardens (St. Andrews) et le Niagara Butterfly Conservatory (Niagara Falls).

Des producteurs horticoles ont également emboité le pas en créant des jardins publics, notamment Cullen Gardens (Scarborough) et Brickmans Botanicals (Sebringville). Le succès d'événements horticoles, comme les Floralies internationales de Québec, a montré l'intérêt populaire grandissant pour l'horticulture ainsi que le potentiel de telles manifestations en matière de tourisme. La Route des Fleurs est même devenue un des principaux produits sur lesquels misent les responsables du tourisme de Laval.

Cette véritable renaissance du jardin se traduit un peu partout en Europe et en Amérique du Nord par des investissements significatifs pour restaurer ou créer des jardins, par la mise en cuvre de programmes d'accès et d'interprétation, de même que par la tenue d'événements destinés à familiariser les visiteurs avec l'art du jardin, à enrichir leur perception, voire à les associer à une création paysagère.
C'est ce dont nous entretiennent A. Reford et A. Hut. Le premier propose un survol du jardin touristique québécois, une ressource exceptionnelle dont il montre d'emblée la richesse, mais aussi la fragilité ; une richesse que confirme incidemment l'ouvrage « Promenade dans les jardins anciens du Québec s, commenté par Kim Desjardins. A. Reford constate cependant que la mise en valeur de ces jardins reste encore trop souvent embryonnaire, malgré les efforts et les investissements consentis par les responsables. Pourtant, le Québec compte un nombre impressionnant de jardins de tout genre et de toutes les périodes historiques, depuis l'époque coloniale françasise jusqu't aujourd' hui. Pourrait-on s'inspirer d'expériences étrangères en matière de mise en valeur et de promotion ? C'est ce que suggère la réflexion d'André Hut. Celui-ci adopte un point de vue similaire à celui d'Alexander Reford pour présenter la transformation du jardin en produit touristique en Belgique. Il fait découvrir un patrimoine exceptionnel qui est notamment l'objet d'une attention particulière et soutenue des administrations publiques. A. Hut insiste particulièrement sur la dimension eminemment culturelle de cette forme de tourisme où culture, nature et patrimoine se rencontrent et se conjuguent au passé, au présent et au futur.

Le phénomène * Villes et villages fleuris * constituerait en quelque sorte l'autre versant de cette mise en tourisme. Le jardi= nage des espaces de la quotidienneté comme pratique de loisir traduirait une sensibilité qui expliquerait, en d'autres circonstances, la fréquentation en hausse des grandes créations paysagères dont elle s'enrichirait. Normand Cazelais propose un survol des tendances actuelles d'un 
concours provincial lancé en 1958 sous le nom de a Villes, villages et campagnes fleuris du Québec . II montre que les réalisations proposées dans le cadre de ce concours s'articulent souvent à une intention de représentation à laquelle l'autre le voisin et le concitoyen, tout autant que le visiteur - est convié. Si la reconnaissance touristique officielle de ces manifestations et de ces aménagements d'envergure parfois étonnante ne fut pas immédiate, loin s'en faut ; on admet aujourd'hui l'intérêt de certains événements initiés dans le cadre du concours, désormais connu sous l'appellation \& Fleurir le Québec *. $\mathrm{N}$. Cazelais en identifie quelques-uns.

Le jardin suscite l"émotion ; il est le lieu d'expériences esthétiques, expériences qui ne se vivent toutefois pas sur le seul mode contemplatif. Le jardin est une création continue, sans cesse réinterprétée, recommencée, renouveléc. C'est là un des traits distinctifs dụ concours présenté par N. Cazelais. Philippe Poullaouec-Gonidec rappelle explicitement cette dimension de l'art du jardin en insistant sur la recréation du monde à laquelle chaque jardinier procède, de l'horticulteur amateur le plus humble au plus grand architecte paysagiste. Cette invention est parfois événement. P. Poullaouec-Gonidec présente deux projets qui ont ếté tenus dans le cadre de la Foire-exposition de Niort et du Festival international des jardins de Chaumont-sur-Loire. Il insiste sur le fait que l'un et l'autre confrontent le visiteur à des creations qui visent fondamentalement à susciter l'étonnement et à révéler des sensibilitês perdues. C'est là manière d'être au monde et d'enrichir un héritage millénaire.

La perte de sensibilitế a laquelle tentent de remédier de tels événements est parfois amnésie des significations. Verity Walker le montre dans un texte où sont comparées les difficultés de la mise en valeur des jardins anciens - en l'occurrence Stowe Landscape Garden - et des paysages régionaux, soit ceux de la région de Charlevoix. Elle constate que l'appréciation n'est trop souvent que superficielle, dans la mesure où le visiteur ne parvient pas à décoder le lieu et à comprendre les aspirations, les motivations et les références de ses concepteurs ou de ses producteurs. L'auteure signale d'ailleurs qu'il existe souvent un décalage important entre la culture des responsables de lieux historiques et celle des visiteurs, ce qui interdit un partage efficace d'une connaissance graduellement constituée. V. Walker milite done pour ce que nous pourrions qualifier de véritable didactique de la fréquentation. Ce faisant, elle rappelle certaines différences qui interdisent d'assimiler entièrement jardins et paysages.

Judith M. LaBelle fait aussi reférence aux paysages de Charlevoix. Tout comme V. Walker, cette dernière a fait partie de l'équipe de personnes-ressources de différents pays réunies en octobre 1998 par les États généraux du paysage québécois, en collaboration avec le Centre d'études collégiales de Charlevoix et le Glynwood Center de New York, afin de réfléchir sur le rôle des paysages eu égard au développement régional et à la mise en valeur touristique'. Le compte-rendu de l'exercice proposé par J.M. LaBelle montre que l'intếrêt pour les paysages pose d'emblée le problème de l'articulation des pré́occupations pour le développement et de celles pour la conservation. Dans ce contexte, la démarche de planification concertante initice s'articule à un regard renouvele, à la fois sur le paysage comme lieu de mémoire et ressource et sur les acteurs comme producteurs de cente ressource, mais aussi comme dépositaires de cette mémoire.

De tels exercices sont devenus pratique courante, notamment en France, où la charte paysagère est en voie de devenir un outil privilégié d'ạmếnagement intercommunal. On lira avec intérết le commentaire de Martin Joly sur un ouvrage récent consacre justement à la charte paysagère et destiné aux acteurs locaux et régionaux du développement.

Cette ouverture sur le paysage ne relève pas d'une confusion des genres ; elle n'est pas fortuite. Tous les auteurs du présent numéro sur les jardins du tourisme y font référence. L'engouement actuel pour les jardins est d'ailleurs inséparable de la quête de paysage qui caractérise nos sociétés. P. Poullaouec-Gonidec propose incidemment, en première partie de son texte, une réflexion sur les arrimages conceptuels et spatiaux qui unissent l'un à l'autre. Jean Decarie transpose en quelque sorte cette réflexion à la ville et sur l'arbre, dont il examine la place et le rôle d'un point de vue historique. La reconstitution du $\alpha$ parcours $*$ de l'arbre en ville et de ses mutations conséquentes — de l'arbre solitaire à la ceinture verte d'agglomération, en passant par le jardin clos et le parc a industriel $*$ - l'amène à poser la problémati- que postindustrielle du parc-paysage intégral, territoire et ressource du tourisme urbain.

Les retombées ếconomiques du tourisme sont désormais considérables, ainsi que le rappellent Julie Jacques et Michel Zins. De là à tenter de mesurer la valeur économique des paysages, il n'y avait qu'un pas que les auteurs ont franchi. J. Jacques ei M. Zins signalent toutefois que si cette valeur ne fait aucun doute, elle reste difficile à déterminer, faute d"une méthode appropriée et universelle. Aussi proposentils des avenues indirectes d'évaluation, dont le coût des investissements compensatoires et le potentiel attractif direct (déterminé par les motivations du choix des destinations) et indirect (le paysage comme facteur d'ambiance).

Mais, avant d'être un bien économique, le jardin est une production anthropologique. Gérard Beaudet propose d'ignorer temporairement les caractères concrets, apparents, aussi bien que la fonction première des jardins pour s'intéresser à leur fonction seconde. Cette mise entre parenthèses des attributs concrets et des fonctions utilitaires lui permet de montrer que le jardin, tout comme les paysages, les établissements agricoles des gentemen farmers, les campagnes patrimonialisées et les a beaux villages $\%$, relèvent de mêmes investissements de valeur dans les formes sensibles de l'espace. Or le parcours génératif qu'il reconstitue montre que ces investissements se présentent avec une intensitế variable, au grế de la transformation de nos sociétés et de l'importance relative de la transcendance et du décret comme modes de disposition des valeurs anthropologiques. Ce faisant, il suggère que le jardin ne crée pas la valeur des lieux ainsi aménagés, mais révèle plutôt un investissement positionnel préalable. II propose en quelque sorte une géographie de notre rapport au monde qui soustend les créations paysagères comme autant de façons d'exprimer ce rapport.

Bienvenue dans les jardins du tourisme.

1 Pour un portrait de cette région fréquentếe par les villegiateurs et les touristes depuis la fin du XVII" siecle, on consultera le numero que lui consacrait Téoros au printemps 1998 (vol, 17, $\mathrm{n}^{*} 1$ ). 